\title{
P21-activated protein kinase 1 is overexpressed in gastric cancer and induces cancer metastasis
}

\author{
LIANG-HUI LI ${ }^{1}$, QI LUO $^{1}$, MIN-HUA ZHENG ${ }^{2-4}$, CHAO PAN ${ }^{5}$, GUO-YANG WU ${ }^{1}$, \\ YI-ZHUO LU ${ }^{1}$, BO FENG ${ }^{2,4}$, XUE-HUA CHEN ${ }^{2,3}$ and BING-YA LIU ${ }^{2,3}$ \\ ${ }^{1}$ Department of General Surgery, Zhongshan Hospital, Xiamen University, Xiamen 361004; \\ ${ }^{2}$ Department of General Surgery, Ruijin Hospital, Shanghai Jiaotong University School of Medicine; \\ ${ }^{3}$ Shanghai Institute of Digestive Surgery; ${ }^{4}$ Shanghai Minimally Invasive Surgery Center, Shanghai 200025; \\ ${ }^{5}$ Department of Pathology, Zhongshan Hospital, Xiamen University, Xiamen 361004, P.R. China
}

Received September 27, 2011; Accepted December 5, 2011

DOI: $10.3892 /$ or.2012.1664

\begin{abstract}
P21-activated protein kinase (Pak1), a main downstream effector of small Rho GTPases, plays an important role in the regulation of cell morphogenesis, motility, mitosis and angiogenesis. However, the role of Pak1 in gastric cancer metastasis remains unclear. Here, we showed that Pak1 is overexpressed in gastric cancer tissues from 74 patients by immunohistochemistry. Overexpression of Pak1 was associated with metastasis and prognosis of gastric cancer. In addition, overexpression of Pak1 increased gastric cancer cell motility and invasion, whereas downregulation of Pak1 expression reduced gastric cancer cell migration and invasion. In further study, data showed that activated Pak1 inhibited stress fiber and focal adhesion complex formation in gastric cancer cells and led to the formation of motile phenotypes. Importantly, activated Pak1 elicited phosphorylation of the ERK and JNK-dependent pathway in gastric cancer cell lines. In conclusion, our results suggest that Pak1 is overexpressed in gastric cancer and plays an important role in the metastasis of gastric cancer. The mechanism by which Pak1 induces cancer metastasis may involve activation of ERK and JNK.
\end{abstract}

\section{Introduction}

Gastric cancer is one of the most common type of cancer worldwide, accounting for the second leading cause of cancerrelated death (1). Studies have shown that abnormal expression of oncogenes, tumor suppressor genes and cell cycle control genes is involved in the evolution of gastric cancer, such as myc, erbB and p53 (2-4). However the exact mechanism of progression and metastasis of gastric cancer has not yet been

Correspondence to: Dr Liang-Hui Li, Department of General Surgery, Zhongshan Hospital, Xiamen University, Xiamen 361004, P.R. China

E-mail: eyelen@sina.com

Key words: gastric cancer, metastasis, $\mathrm{P} 21$-activated protein kinase, ERK, JNK clarified. A better understanding of molecular changes during stomach carcinogenesis may be significant in gastric cancer research.

p21-activated kinase $1(\mathrm{Pak} 1)$ is the best characterized member of an evolutionary conserved family of serine/ threonine kinases (5), which plays a role in a variety of cellular functions such as cytoskeletal reorganization, cell motility, apoptosis and transformation (6-8). Pak1 has been identified as an effector molecule for the small GTPases Rho, Racl and Cdc42 (9). In addition, other signaling molecules can also affect Pak1 activity, including AKT, JAK2, PDK1 and PKA (10-13). Activation of Pak1 leads to autophosphorylation at several sites, including threonine-423 within the activation loop of the kinase (14).

Amplification of Pak1 has been found in breast cancer, renal cancer and liver cancer. Moreover, Pak1 has been reported to induce proliferation, motility and invasion of these cancer cells by being involved in several cell signaling pathways, such as mitogen-activated protein kinases (MAPK) and nuclear factor- $\kappa \mathrm{B}(\mathrm{NF}-\kappa \mathrm{B})(8,15,16)$. Recently, we reported that Pak1 was also overexpressed in colorectal cancer and activated Pak1 regulated colorectal cancer metastasis requiring an ERK-dependent phosphorylation of FAK at Ser-910 (17). Nevertheless, the exact molecular mechanism by which Pak1 contributes to gastric cancer metastasis still remains unclear. In this study, we examined the expression of Pak1 in human gastric cancer and characterize its roles in gastric cancer metastasis. Our data indicated that Pak1 expression was significantly up-regulated in human gastric cancer. This overexpression was associated with more aggressive tumor behavior, and clinical follow-up showed that increased Pak-1 expression and activity were related to reduced patient survival. Ectopic expression of Pak1 obviously increased the motility and invasion of gastric cancer cells, while down-regulation of Pak1 expression or activity impaired the motility and invasion of gastric cancer cells. Moreover, Pak1 might affect metastasis of gastric cancer cells by phosphorylation of Erk and JNK signaling pathway. Our results present a novel mechanism for Pak1 regulation of human gastric cancer cell metastasis, which may be helpful to provide a therapeutic strategy for gastric cancer. 


\section{Materials and methods}

Tissue samples and cell culture. Paired samples of primary gastric cancer and the corresponding non-tumorous gastric tissue were obtained from the Xiamen University Zhongshan Hospital (Xiamen, China). Specimens were formalin-fixed and paraffin-embedded. The study protocol was approved by the Medical Ethics and Human Clinical Trial Committee at Xiamen University Zhongshan Hospital. MKN45 human gastric cancer cell was maintained in RPMI-1640 medium containing $10 \%$ fetal bovine serum (FBS).

Materials. Anti-Pak1 (N-20) (sc-882), anti-phospho-Pak1 (Thr423) (sc-12925) and anti-DsRed (L-18) (sc-33353) antibodies were obtained from Santa Cruz Biotechnology (Santa Cruz, CA, USA). Anti-JNK, anti-phospho-JNK, anti-p42/44MAPK, anti-phospho-p42/44MAPK, anti-p38MAPK and anti-phosphop38MAPK antibodies were from Cell Signaling Technology (Beverly, MA, USA). Anti-Rac1 and anti-Cdc42 antibodies were from BD Biosciences (Palo Alto, CA, USA). Alexa Fluor-488 phalloidin was from Molecular Probes (Invitrogen Detection Technologies). Anti-paxillin antibody (Clone 5H11) was from Labvision (Neomarkers). Anti-mouse IgG FITC conjugate antibody was from Sigma (St. Louis, MO, USA). Anti-glyceraldehyde-3-phosphate Dehydrogenase (GAPDH) antibody was from KangChen.

Constructs and production of stable cell line. The Pak1 construct, pDs-Red2-Pak1 was a generous gift from Dr Jonathan Chernoff (Fox Chase Cancer Centre, Philadelphia, PA, USA). For constructing the stable transfectant, pDs-Red2 and pDs-Red2-Pak1 were separately transfected into MKN45 human gastric cancer cell by using the Lipofectamine 2000 reagent, according to the manufacturer's instructions (Invitrogen, Carlsbad, CA, USA). The transfected cells were selected in growth medium containing $1000 \mu \mathrm{g} / \mathrm{ml}$ Geneticin (G418; Life Technologies, Grand Island, NY, USA). After 4-8 weeks, individual cell colonies were transferred for clone expansion and maintained in culture medium supplemented with $600 \mu \mathrm{g} / \mathrm{ml} \mathrm{G} 418$.

Pakl siRNA. For RNA interference (RNAi) of Pak1, Pak1 small interfering RNA and control siRNA were purchased from Shanghai GenePharma Co., Ltd. Each of them (100 pmol in $2 \mathrm{ml}$ medium) was transfected into MKN45 human gastric cancer cell by using Lipofectamine 2000 (Invitrogen).

Cell motility and invasion assay. The polycarbonate membranes $6.5 \mathrm{~mm}$ in diameter with $8 \mu \mathrm{m}$ pores (Corning Costar, New York, NY, USA) coated with Matrigel (BD Biosciences, Bedford, MA, USA) were used for invasion assay, whereas the membranes without Matrigel were for motility assay. After adding medium containing $10 \%$ FCS to the bottom chambers, single-cell suspensions in medium containing $0.1 \%$ BSA were seeded onto the filters $\left(1 \times 10^{5}\right.$ cells per each well) and incubated for 24 or $48 \mathrm{~h}$ at $37^{\circ} \mathrm{C}, 5 \% \mathrm{CO}_{2}$. Filters were then washed and cells on the upper surface were removed with cotton swabs. The cells that had migrated or invaded to the lower surface of the filter inserts were fixed with 5\% paraformaldehyde for $15 \mathrm{~min}$ and stained with $0.1 \%(\mathrm{w} / \mathrm{v})$ Crystal violet for $15 \mathrm{~min}$. The number
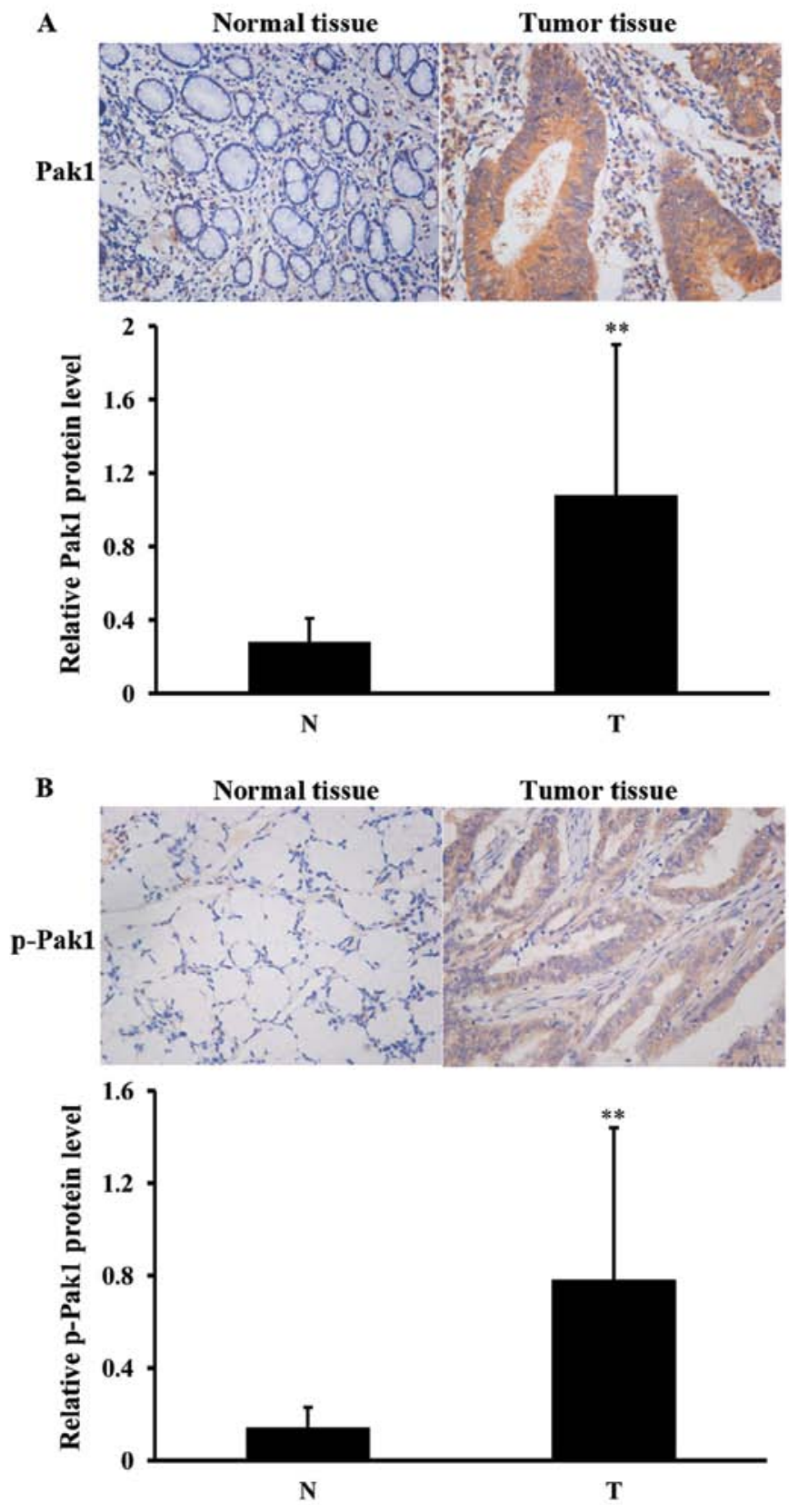

Figure 1. Imunohistochemical demonstration of Pak1 and phosphorylated Pak1 in human gastric cancer. (A) Stronger staining of Pak1 was located in the cytoplasm of gastric cancer, while very weak staining was observed in non-tumorous (normal) tissue. Statistical analysis with relative expression level of Pak1 in 74 of paired samples of gastric cancer and normal tissues. ${ }^{* *} \mathrm{P}<0.001$ (bottom). (B) Stronger staining of phosphorylated Pak1 was located in the cytoplasm of gastric cancer, while very weak staining was observed in normal tissue. Statistical analysis with relative expression level of phosphorylated Pak1 in 74 of paired samples of gastric cancer and normal tissues. ${ }^{* *} \mathrm{P}<0.001$ (bottom). Original magnifications, $\mathrm{x} 200$ (top).

of migrated or invaded cells was microscopically counted and 3 independent experiments were carried out to get an average cell number per high magnification field.

Immunofluorescence assays and confocal microscopy. Cells were treated with lysophosphatidic acid (LPA) for $15 \mathrm{~min}$, fixed in $4 \%$ para-formaldehyde for $5 \mathrm{~min}$, and permeabilized with $0.1 \%$ Triton in PBS for 3 min. Alexa Fluor-488 phalloidin was used to visualize F-actin containing structures. Anti-paxillin 

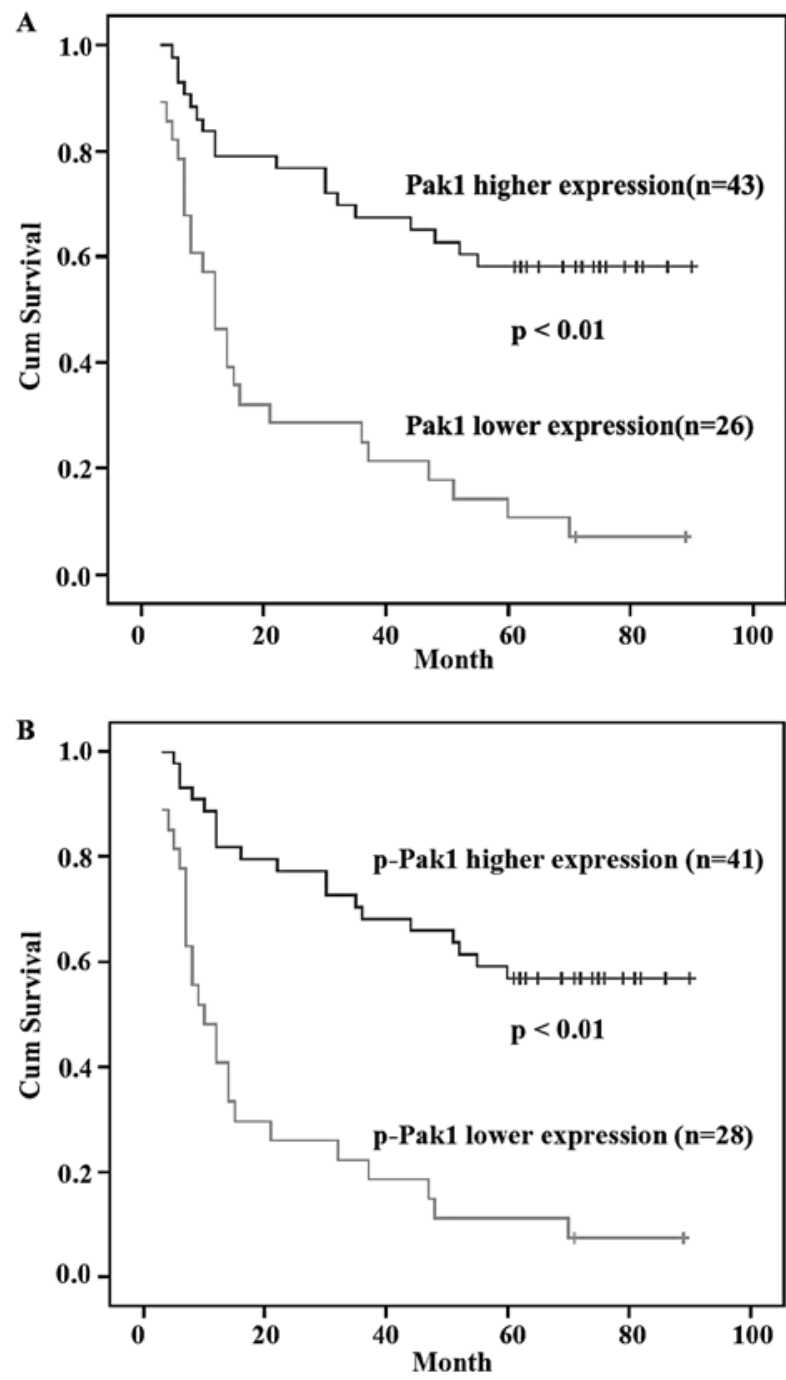

Figure 2. Kaplan-Meir survival curves of Pak1 lower-expression group and Pak1 higher-expression group (top). Kaplan-Meir survival curves of phosphorylated Pak1 lower-expression group and phosphorylated Pak1 higher-expression group (bottom).

combined with anti-mouse IgG FITC conjugate antibody were used to show focal adhesion complex. Immunofluorescence imaging was captured in aZeissLSM510 laser scanning confocal microscope (Carl Zeiss Microimaging Inc., Thornwood, NY, USA).

Western blot analysis. Cells were harvested into RIPA lysis buffer (Pierce) with freshly added protease inhibitor cocktail (Roche) and phosphatase inhibitor cocktail (Roche). The cell lysate was cleared by centrifugation at $4^{\circ} \mathrm{C}$ and the supernatant was stored in small aliquots at $-80^{\circ} \mathrm{C}$. The $20 \mu \mathrm{g}$ sample was loaded into each lane, separated by SDS -PAGE, transferred to polyvinylidene difluoride membrane, and probed with respective antibodies.

Immunohistochemistry. Sections were deparaffinized in xylene and hydrated through a graded series of alcohol to distilled water. Endogenous peroxidase was blocked by incubation in $0.3 \% \mathrm{H}_{2} \mathrm{O}_{2}$ in methanol for $30 \mathrm{~min}$. Antigen retrieval was performed by microwave irradiation in $0.01 \mathrm{M}$ citrate buffer, pH 6.0. Following washing 3 times with $1 \times$ PBS, nonspecific

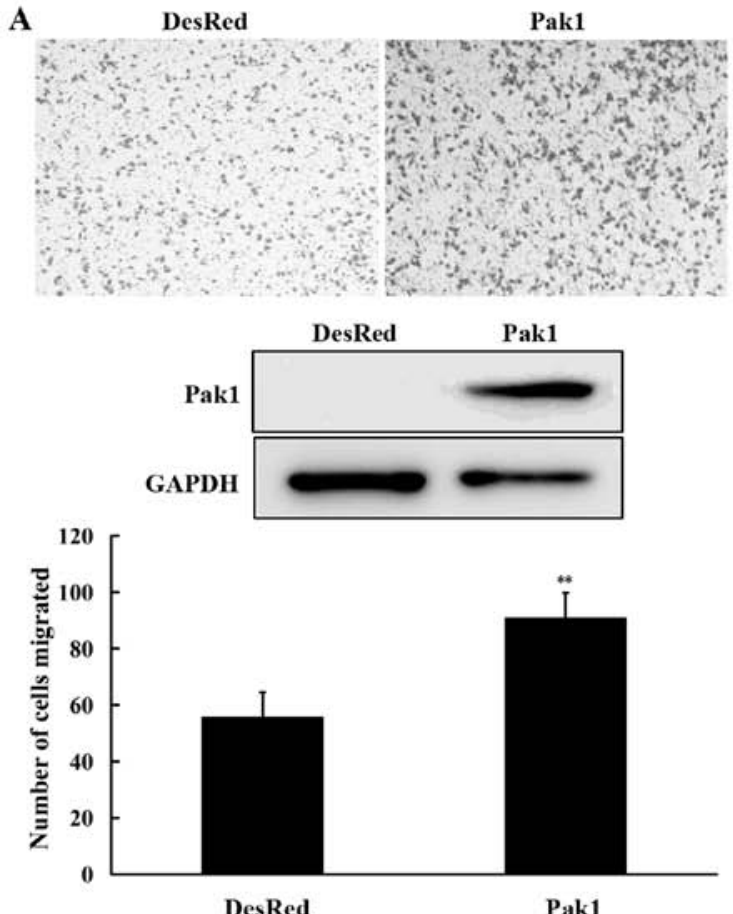

B
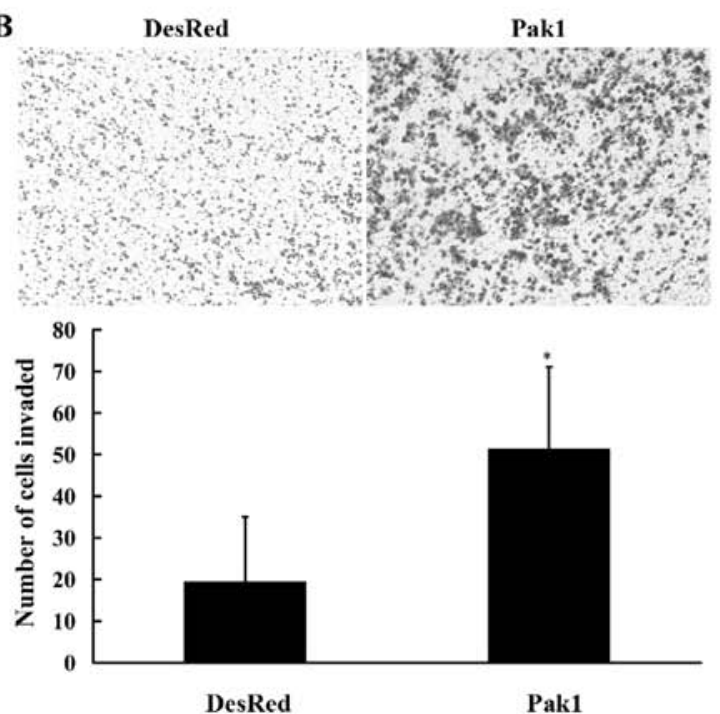

Figure 3. Pak1 induced gastric cancer cell migration and invasion. (A) Representative images showed MKN45-DsRed2 and MKN45-Pak1 cells to migrate for $24 \mathrm{~h}$ (top). Expression of DsRed-Pak1 in MKN45 cells was confirmed by anti-DsRed antibody (insert). Migrated cells of 6 randomly selected fields were counted. ${ }^{* *} \mathrm{P}<0.01$ (bottom). (B) Representative images showed MKN45-DsRed2 and MKN45-Pak1 cells to invade for $48 \mathrm{~h}$ (top). Invaded cells of 6 randomly selected fields were counted. ${ }^{*} \mathrm{P}<0.05$, (bottom).

binding was blocked with 5\% NGS in $1 \mathrm{X}$ TBS for $1 \mathrm{~h}$. To detect Pak1 expression, sections were incubated overnight at $4^{\circ} \mathrm{C}$ with anti-Pak1 specific polyclonal antibody (N-20 Santa Cruz Biotechnology) at a 1: 50 dilution. Phosphorylated Pak1 was detected by a polyclonal anti-Pak1 phosphospecific antibody (sc-12925, Santa Cruz Biotechnology) at a 1:150 dilution. Antibody binding was localized using a biotinylated secondary antibody, avidin-conjugated HRP and DAB substrate. Slides were counterstained with hematoxylin and eosin. When viewed under a light microscope, positive cells stained brown. The 
Table I. Association of Pak1 expression and activation with clinicopathological features in human gastric cancer.

\begin{tabular}{|c|c|c|c|c|c|}
\hline $\begin{array}{l}\text { Clinicopathological } \\
\text { features }\end{array}$ & $\mathrm{n}$ & $\begin{array}{c}\text { Pak1 } \\
(\text { mean } \pm \mathrm{SD})\end{array}$ & $\mathrm{p}$-value & $\begin{array}{c}\mathrm{p}-\mathrm{Pak} 1 \\
(\mathrm{mean} \pm \mathrm{SD})\end{array}$ & p-value \\
\hline \multicolumn{6}{|l|}{ Age } \\
\hline$>60$ years & 40 & $1.26 \pm 0.68$ & \multirow[t]{2}{*}{0.462} & $0.79 \pm 0.86$ & \multirow[t]{2}{*}{0.501} \\
\hline$\leq 60$ years & 34 & $1.28 \pm 0.59$ & & $0.77 \pm 0.92$ & \\
\hline \multicolumn{6}{|l|}{ Gender } \\
\hline Male & 61 & $1.25 \pm 0.71$ & \multirow[t]{2}{*}{0.709} & $0.77 \pm 1.03$ & \multirow[t]{2}{*}{0.798} \\
\hline Female & 13 & $1.24 \pm 0.88$ & & $0.78 \pm 1.01$ & \\
\hline \multicolumn{6}{|l|}{ Depth of invasion } \\
\hline $\mathrm{T} 1$ & 6 & $0.55 \pm 0.41$ & \multirow[t]{4}{*}{0.013} & $0.33 \pm 0.47$ & \multirow[t]{4}{*}{0.015} \\
\hline $\mathrm{T} 2$ & 24 & $0.88 \pm 0.91$ & & $0.59 \pm 0.88$ & \\
\hline $\mathrm{T} 3$ & 36 & $1.19 \pm 1.18$ & & $0.88 \pm 1.77$ & \\
\hline $\mathrm{T} 4$ & 8 & $1.32 \pm 1.27$ & & $1.01 \pm 1.73$ & \\
\hline \multicolumn{6}{|l|}{ Lymph node status } \\
\hline No & 18 & $0.49 \pm 0.87$ & \multirow[t]{4}{*}{0.000} & $0.33 \pm 0.48$ & \multirow[t]{4}{*}{0.019} \\
\hline N1 & 23 & $0.74 \pm 0.59$ & & $0.61 \pm 0.53$ & \\
\hline $\mathrm{N} 2$ & 19 & $1.05 \pm 0.63$ & & $0.87 \pm 0.92$ & \\
\hline $\mathrm{N} 3$ & 14 & $1.29 \pm 1.59$ & & $1.10 \pm 1.38$ & \\
\hline \multicolumn{6}{|l|}{ Distant metastasis } \\
\hline M0 & 58 & $0.87 \pm 0.77$ & \multirow[t]{2}{*}{0.014} & $0.54 \pm 0.68$ & \multirow[t]{2}{*}{0.007} \\
\hline M1 & 16 & $1.25 \pm 0.80$ & & $1.02 \pm 0.92$ & \\
\hline \multicolumn{6}{|l|}{ Degrade } \\
\hline I & 9 & $0.72 \pm 0.53$ & \multirow[t]{3}{*}{0.027} & $0.41 \pm 0.86$ & \multirow[t]{3}{*}{0.002} \\
\hline II & 54 & $0.99 \pm 0.89$ & & $0.68 \pm 0.67$ & \\
\hline III & 11 & $1.29 \pm 0.75$ & & $1.05 \pm 1.08$ & \\
\hline \multicolumn{6}{|l|}{ Stage } \\
\hline I & 6 & $0.59 \pm 0.07$ & \multirow[t]{4}{*}{0.000} & $0.40 \pm 0.22$ & \multirow[t]{4}{*}{0.005} \\
\hline II & 21 & $0.88 \pm 0.37$ & & $0.68 \pm 0.79$ & \\
\hline III & 31 & $1.13 \pm 0.59$ & & $0.85 \pm 0.87$ & \\
\hline IV & 16 & $1.47 \pm 1.01$ & & $1.18 \pm 1.12$ & \\
\hline
\end{tabular}

semi-quantitative evaluation of stain was according to Carter et al (18).

Statistical analysis. Fisher's exact or $\chi^{2}$ test was used for statistical analysis of categorical data. The cancer-specific survival outcome was expressed by applying the KaplanMeier method, and the log-rank test was used to compare the prognostic significance of individual variables on survival. Statistical SPSS Version 15.0 was used to analyze data. A p-value of $<0.05$ was considered statistically significant.

\section{Results}

Pakl expression and activation correlated with clinicopathologic factors of human gastric cancer. We determined the protein expression level of Pak1 and phosphor-Pak1 in gastric cancer tissues and matched adjacent non-cancerous tissues by immunohistochemical staining. Positive staining was observed in the cytoplasm of gastric epithelial cells. Compared with matched non-tumorous tissues, 78.5\% (58 of 74) of the gastric cancer samples were found to have a higher expression of Pak1 (>2-fold) and $70.3 \%$ (52 of 74) of the gastric cancer samples to have a higher expression of activated Pak1 (>2-fold). Overall, significantly stronger staining of Pak1 and activated Pak1 was observed in gastric cancer than in non-tumorous tissues $(1.08 \pm 0.82$ vs. $0.28 \pm 0.13$ and $0.78 \pm 0.66$ vs. $0.14 \pm 0.09, \mathrm{p}<0.01$ and $\mathrm{p}<0.01$, Fig. 1). To further elucidate the correlation between expression level and activation of Pak1 and clinicopathological factors in human gastric cancer, 74 malignant tumors were further analyzed. The result showed that Pak1 expression and phosphorylation in tumor tissue were significantly associated with the depth of invasion ( $\mathrm{p}=0.013$ and $\mathrm{p}=0.015)$, lymph node status $(\mathrm{p}<0.01$ and $\mathrm{p}=0.019)$, distant metastasis $(\mathrm{p}=0.014$ and $\mathrm{p}=0.007)$, tumor stages $(\mathrm{p}=0.027$ and $\mathrm{p}=0.002)$ and tumor grades $(\mathrm{p}<0.01$ and $\mathrm{p}=0.005)$. Nevertheless, Pak1 expression and phosphorylation were independent of tumor location, gender and age (Table I). Moreover, clinical follow-up was available for all but 5 cancer cases in this study and indicated 


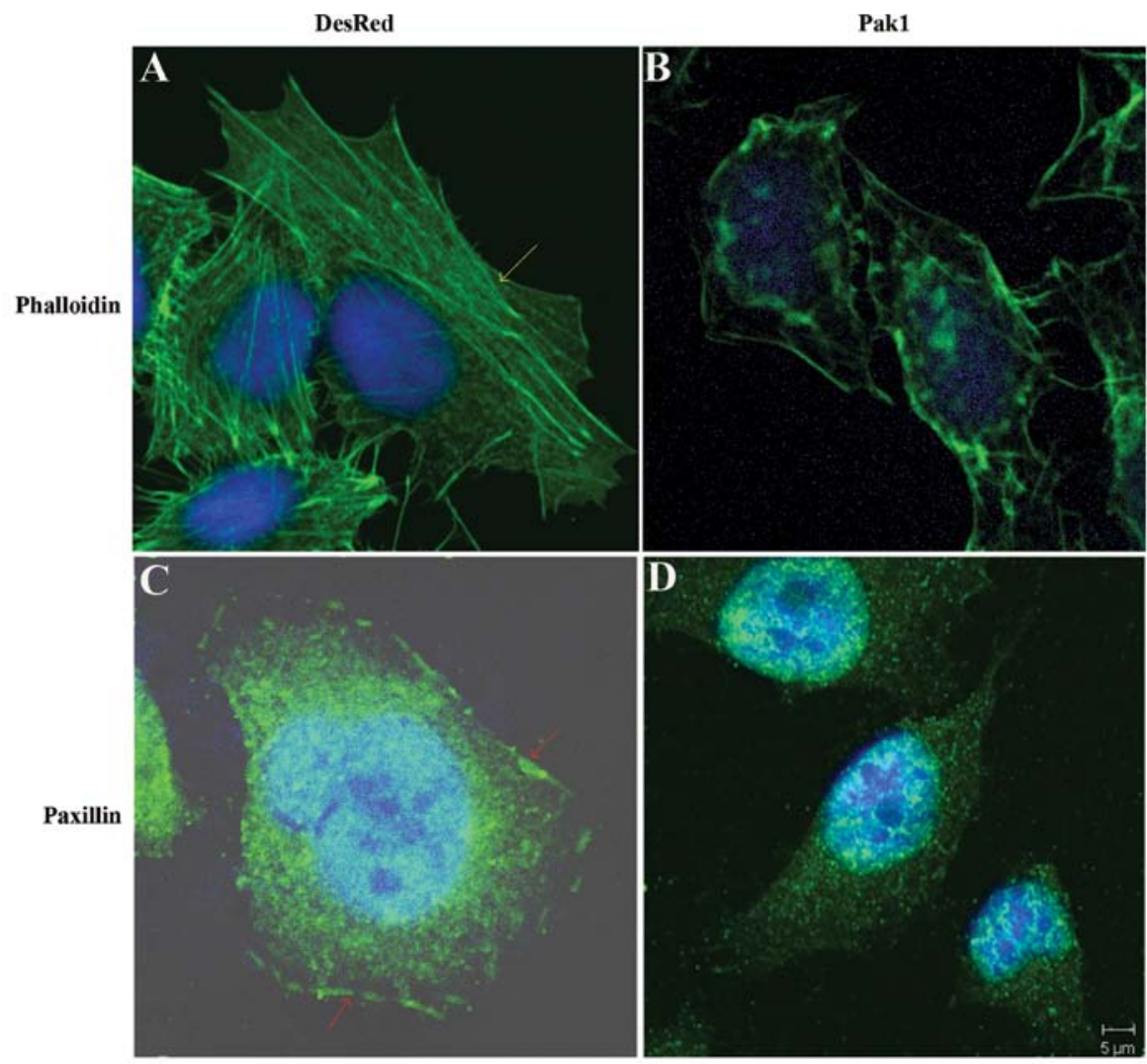

Figure 4. Pak1 regulation of cytoskeleton reorganization of gastric cancer cells. (A and B) The representative confocal images showed the staining of F-actin containing structures in MKN45-DsRed2 cells and MKN45-Pak1 cells with Alexa Fluor-488 phalloidin. Yellow arrow indicate filopodia. (C and D) The representative confocal images show staining of focal adhesion complex in MKN45-DsRed2 cells and MKN45-Pak1 cells with anti-paxillin antibody. Bar, $5 \mu \mathrm{m}$

that the 5-year survival rate of the lower-Pak1 expression and phosphorylation group were significantly better than that of the higher group (62.5 vs. $21.7 \%$ and 62.2 vs. $23.8 \%, p<0.01$ and $\mathrm{p}<0.01)$. The lower-Pak1 expression and phosphorylation group were defined as those at or below the median value (1.14 and 0.84 , respectively), whereas the higher group consisted of patients above the median value (Fig. 2).

Overexpression of Pakl enhanced cell migration and invasion. To elucidate the effects of Pak1 in gastric cancer, MKN45 gastric cancer cells were used to establish stable cell line overexpressing the pDs-Red 2 fusion form of Pak1. As shown in Fig. 3A, the stable transfectant cell line MKN45-Pak1 was established and Pak1 expression was significantly up-regulated compared with the mock transfectant with pDsRed2 (named MKN45-DsRed2). To test whether Pak1 affects the motility of gastric cancer cells, the migration assay was performed with MKN45-DsRed2 cells and MKN45-Pak1 cells. We found that up-regulation of Pak1 expression enhanced cell migration compared with MKN45-DsRed2 (91.00 \pm 8.80 vs. $55.80 \pm 8.73$, p $<0.01 \mathrm{Fig}$. $3 \mathrm{~A}$ ). Then, the invasion assay was done and we observed that ectopic expression of Pak1 resulted in an increased invasion of MKN45 cells compared with the control $(51.40 \pm 19.67$ vs. $19.40 \pm 15.67$, $\mathrm{p}=0.02$ Fig. $3 \mathrm{~B})$.

Activation of Pakl down-regulated stress fiber and focal adhesion complex formation. We tested if Pak1 regulated the reorganization of cytoskeleton components to increase the migration and invasion of gastric cancer cells. As revealed by phalloidin staining, much fewer stress fibers were formed in the Pak1 stable clone than the control (Fig. 4A and B). As revealed by paxillin staining, the focal adhesion complexes were fewer in the Pak1 stable clone than the control (Fig. 4C and D). These results suggest that Pak1 enhances migration and invasion of gastric cancer cells by down-regulating stress fibers and focal adhesion complex formation.

Knockdown of endogenous Pakl by siRNA reduces cell migration and invasion. To confirm the role of Pak1 on cell migration and invasion, we used RNAi to specifically knock down endogenous Pak1 in MKN45 cells. The specific knockdown of endogenous Pak1 in MKN45 cells was confirmed with immunoblotting (Fig. 5A). We observed that cells transfected with Pak1 siRNA migrated much more slowly than those treated with control siRNA(77.00 \pm 38.66 vs. $306.60 \pm 58.53$, $\mathrm{p}<0.01$, Fig. 5A). Similarly, cells transfected with Pak1 siRNA invaded much more slowly than the control $(6.00 \pm 6.28$ vs. $152.00 \pm 110.51, \mathrm{p}<0.01$, Fig. $5 \mathrm{~B})$, suggesting that the knockdown of Pak1 retarded the migration and invasion of gastric cancer cells.

Activation of ERK1/2 and JNK was required for Pakl-mediated migration and invasion of gastric cancer cells. To understand how Pak1 induced migration and invasion of gastric cancer cells, we investigated whether the activation of Pak1 regulated its well-defined downstream effectors, JNK, p38MAPK and 

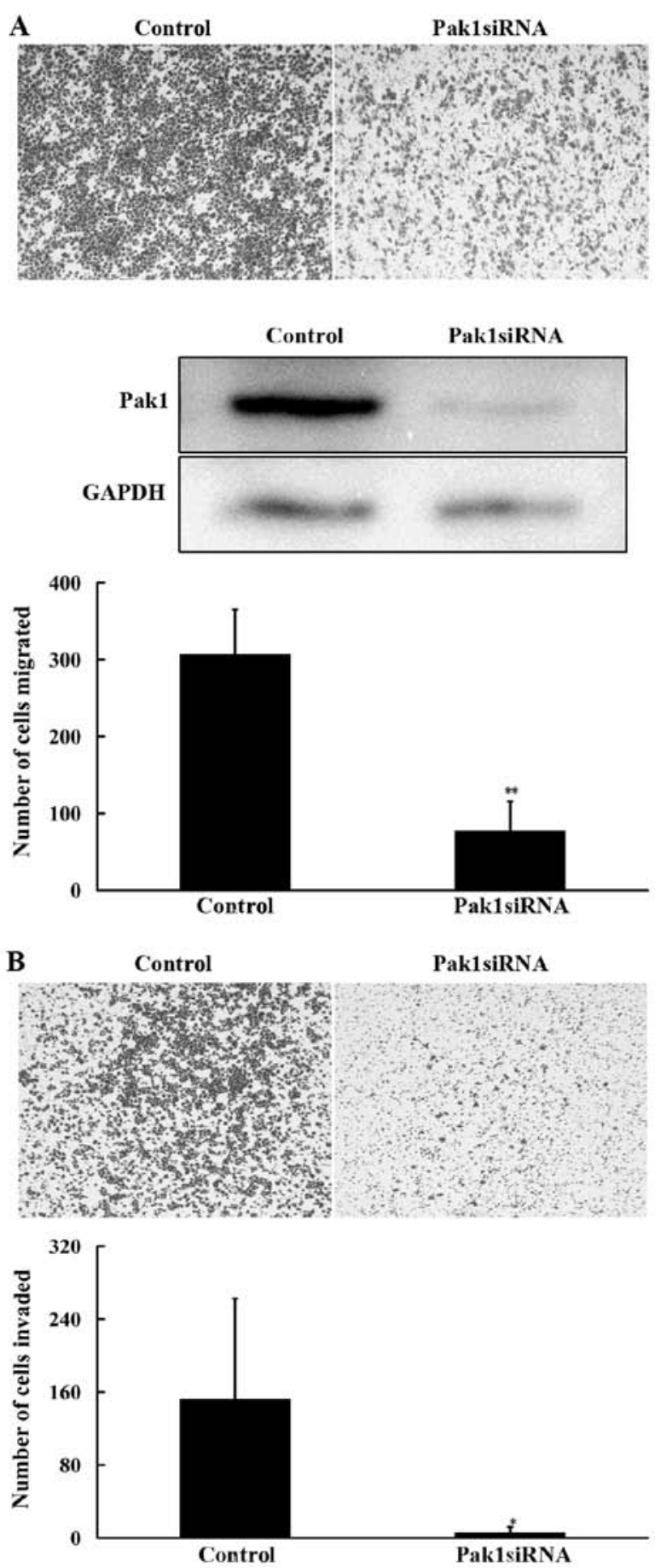

Figure 5. Knockdown of endogenous Pak1 by siRNA reduced cell migration and invasion. (A) Representative images show MKN45 cells transfected with Pak1 siRNA and control siRNA to migrate for $24 \mathrm{~h}$ (top). Knockdown of Pak1 in MKN45 cells by transfected with Pak1 siRNA was confirmed by Western blotting (inset). Migrated cells of 6 randomly selected fields were counted. ${ }^{* *} \mathrm{P}<0.01$ (bottom). (B) Representative images show MKN45 cells transfected with Pak1 siRNA and control siRNA to invade for $48 \mathrm{~h}$ (top). Invaded cells of 6 randomly selected fields were counted. " $\mathrm{P}<0.05$ (bottom).

extracellular signal regulated kinase (ERK). We observed that phosphorylation of Pak1 was obviously increased in Pak1 overexpressed cells compared with control cells, which suggested that the activity of Pak1 may provide an advantage for gastric cancer cells to migrate and invade. $\mathrm{Cdc} 42$

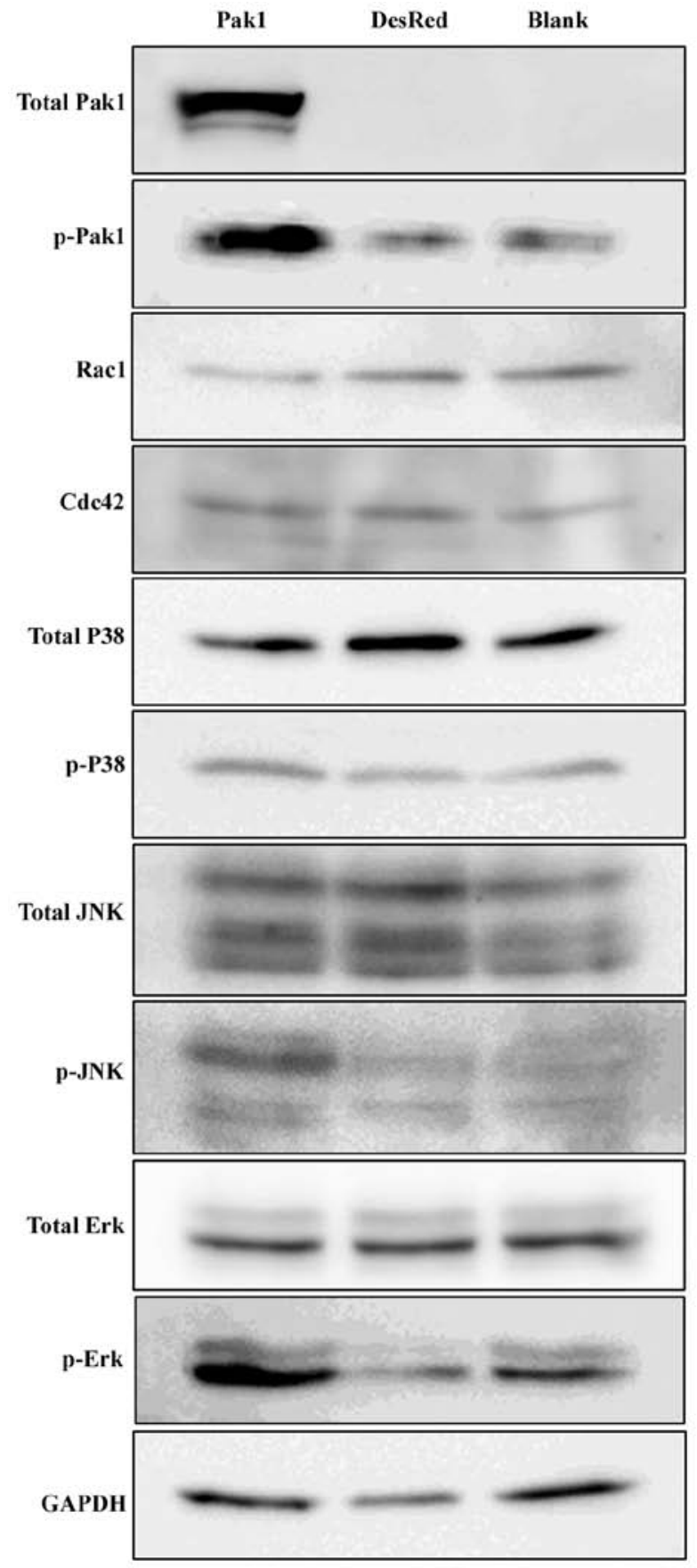

Figure 6. Pak1 regulated human gastric cancer cell migration through ERK and JNK-dependent pathway. Western blot analysis of Pak1, phospho-Pak1 (p-Pak1), Rac1, Cdc42, ERK1/2, phospho-ERK (p-ERK) 1/2, p38MAPK, phospho-p38MAPK, JNK, phospho-JNK (p-JNK) and GAPDH between MKN45, MKN45-DsRed2 and MKN45-Pak1 cells.

and Rac1, upstream regulators of Pak1, however, were not overexpressed in Pak1 stable clone. Both JNK and ERK were remarkably phosphorylated in Pak1 stable clone, whereas p38MAPK was not (Fig. 6). To further determine whether activation of JNK or ERK was required for Pak1-mediated migration and invasion of gastric cancer cells, MKN45-Pak1 cells were treated with U0126, SP600125 and SB203580, respectively. The results showed that migration and invasion of MKN45-Pak1 cells were specifically inhibited by JNK inhibitor SP600125 or ERK Inhibitor U0126, not by p38 MAPK inhibitor SB203580 (Fig. 7). 

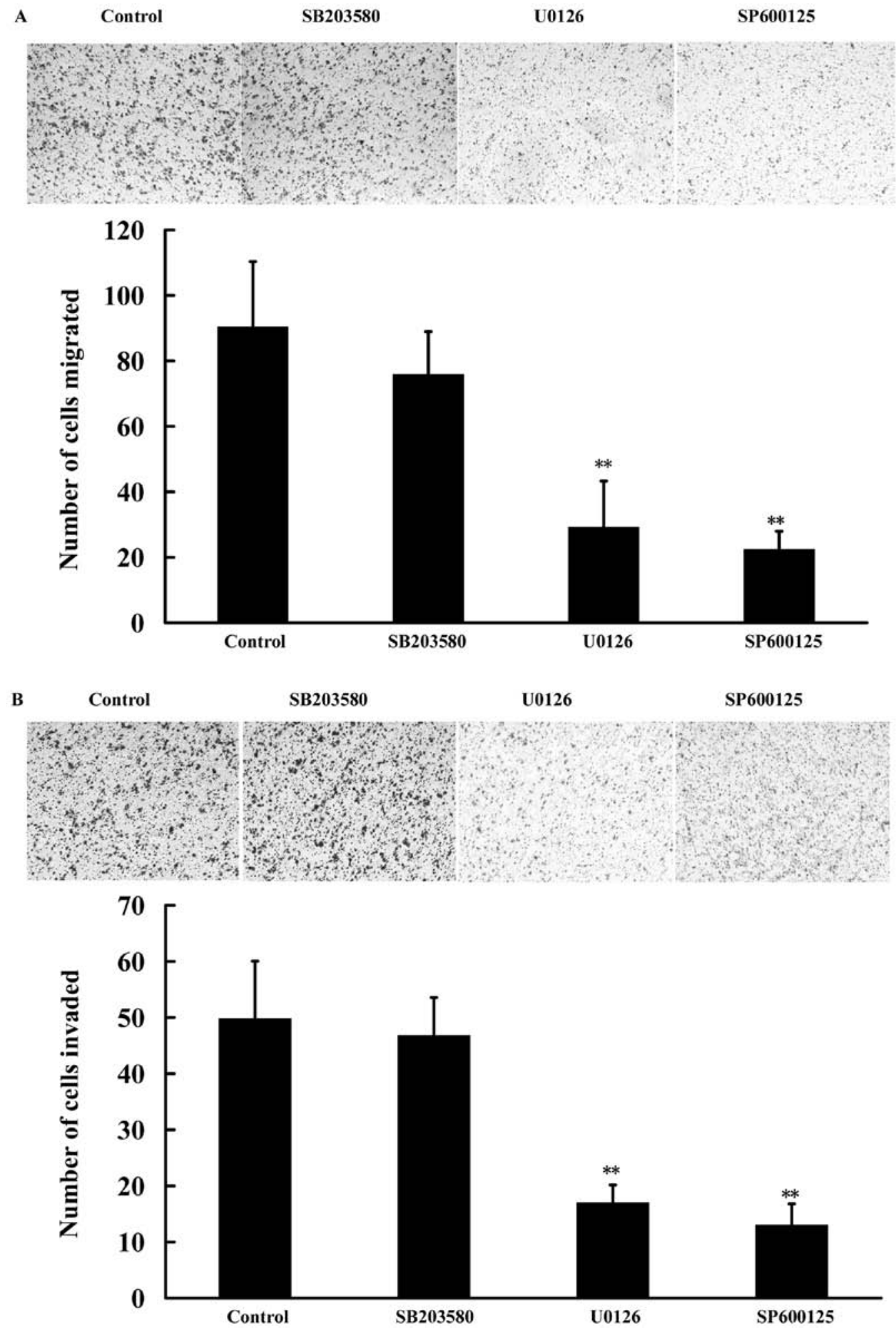

Figure 7. Gastric cancer cell migration and invasion induced by Pak1 were specifically inhibited by JNK inhibitor or ERK inhibitor, not by p38 MAPK inhibitor. (A) MKN45-Pak1 cells were pretreated with pharmacologic inhibitors U0126 (10 $\mu \mathrm{mol} / 1$; ERK inhibitor), SB203580 (2.5 $\mu \mathrm{mol} / 1$; p38 MAPK inhibitor) and SP600125 (50 $\mu \mathrm{mol} / 1$; JNK inhibitor), respectively, and then were allowed to migrate for $24 \mathrm{~h}$ (top). (B) MKN45-Pak1 cells were pretreated with pharmacologic inhibitors (A), and then were allowed to invade for $48 \mathrm{~h}$ (top). (A and B) Quantification of three independent experiments. ${ }^{* *} \mathrm{P}<0.01$, statistical significance between the DMSO control and U0126 or between the DMSO control and SP600125 (bottom).

\section{Discussion}

Although it has been reported that Pak1 overexpression might induce gastric cancer cell proliferation by regulating cyclin B1 expression (19), the studies about the role of Pak1 in invasion and metastasis of gastric cancer were still absent. In our study, both Pak1 overexpression and activity in gastric cancer were observed to significantly correlate with more aggressive tumor behavior in terms of depth of invasion, lymph node metastasis, distant metastasis, tumor stages, tumor grades, and shorter 
disease-free survival. These data demonstrate that both Pak1 overexpression and activity may induce invasion and metastasis of gastric cancer. As expected, we found that gastric cancer cells with Pak1 overexpression had higher migration and invasion rate, whereas those with down-regulation of Pak1 expression had significantly reduced cell migration and invasion rate. It has been reported that cytoskeleton reorganization is a fundamental component of cell motility $(20,21)$. Moreover, Pak1 has been shown to be accompanied by the disassembly of stress fibers and focal adhesion complexes, which is necessary for increased motility $(16,22,23)$.

Our previous data showed that Pak1 affected cytoskeleton reorganization resulting in improving colorectal cancer cell migration (17). Thus, we would like to propose that Pak1 may play a similar role in modulating cytoskeleton reorganization of gastric cancer cells. Consistent with this hypothesis, we observed that the ability of gastric cancer cells to form stress fibers and focal adhesion complex was attenuated by overexpression of Pak1. Accumulating evidence have suggested a role for Pak1 in regulating mitogen activated protein kinase cascades in carcinogenesis $(15,16)$. Our previous data also proved that Pak1 regulates colorectal cancer metastasis requiring ERK1/2 activation (17). To investigate the underlying mechanism, we observed that both ERK and JNK were activated in gastric cancer cells overexpressing Pak1, whereas p38MAPK was not. Moreover, both ERK and JNK-dependent pathway were required in Pak1-mediated gastric cancer cell migration.

The results suggest that Pak1 may influence gastric cancer cell migration by at least two mechanisms. However, the detail mechanisms which Pak1 regulates these two signaling pathways in gastric cancer need further study. Although Pak1 has been identified as the downstream effector target of Rac1 and Cdc42 in mammalian cells (24), no significant difference of these regulators at protein level was observed in our present study. Given that Pak1 is also activated by several other physiological signals, such as guanine nucleotide factor pix (25), filamin A (26), adaptor protein Nck (27) and phosphatidylinositol 3-kinase/Akt (10), activation of Pak1 in gastric cancer cells may be induced through Cdc42/Rac1independent mechanisms.

In conclusion, we have shown that Pak1 plays an important role in gastric cancer metastasis. The mechanism by which Pak1 induces cancer metastasis may involve activation of ERK and JNK.

\section{Acknowledgements}

We thank Dr Hua D. Lu (Department of Pathology, Zhongshan Hospital, Xiamen University) for excellent technical assistance and advice.

\section{References}

1. Tsugane S and Sasazuki S: Diet and the risk of gastric cancer: review of epidemiological evidence. Gastric Cancer 10: 75-83 2007.

2. Nin NI, Yonemura Y, Matsumoto H, Sugiyama K, Kamata T, Miwa K, Miyazaki I and Shiku H: Expression of c-myc gene product in gastric carcinoma. Oncology 48: 149-153, 1991.

3. Falck VG and Gullick WJ: C-erbB-2 oncogene product staining in gastric adenocarcinoma. An immunohistochemical study. J Pathol 159: 107-111, 1989.
4. Yamada Y, Yoshida T, Hayashi K, Sekiya T, Yokota J, Hirohashi S, Nakatani K, Nakano H, Sugimura T and Terada M: p53 gene mutations in gastric cancer metastases and in gastric cancer cell lines derived from metastases. Cancer Res 51: 5800-5855, 1991.

5. Kumar R, Gururaj AE and Barnes CJ: p21-activated kinases in cancer. Nat Rev Cancer 6: 459-471, 2006.

6. Kumar R and Vadlamudi RK: Emerging functions of p21-activated kinases in human cancer cells. J Cell Physiol 193: 133-144, 2002.

7. Schurmann A, Mooney AF, Sanders LC, et al: p21-activated kinase 1 phosphorylates the death agonist bad and protects cells from apoptosis. Mol Cell Biol 20: 453-461, 2000.

8. Vadlamudi RK, Adam L, Wang RA, et al: Regulatable expression of p21-activated kinase-1 promotes anchorage-independent growth and abnormal organization of mitotic spindles in human epithelial breast cancer cells. J Biol Chem 275: 36238-36244, 2000.

9. Narumiya S, Ishizaki T and Watanabe N: Rho effectors and reorganization of actin cytoskeleton. FEBS Lett 410: 68-72, 1997

10. Zhou GL, Zhuo Y, King CC, Fryer BH, Bokoch GM and Field J: Akt phosphorylation of serine 21 on Pak1 modulates Nck binding and cell migration. Mol Cell Biol 23: 8058-8069, 2003.

11. Rider L, Shatrova A, Feener EP, Webb L and Diakonova M: JAK2 tyrosine kinase phosphorylates PAK1 and regulates PAK1 activity and functions. J Biol Chem 282: 30985-30996, 2007.

12. King CC, Gardiner EM, Zenke FT, et al: p21-activated kinase (PAK1) is phosphorylated and activated by 3-phosphoinositidedependent kinase-1 (PDK1). J Biol Chem 275: 41201-41209, 2000.

13. Howe AK and Juliano RL: Regulation of anchorage-dependent signal transduction by protein kinase $\mathrm{A}$ and $\mathrm{p} 21$-activated kinase. Nat Cell Biol 2: 593-600, 2000.

14. Chong C, Tan L, Lim L and Manser E: The mechanism of PAK activation. Autophosphorylation events in both regulatory and kinase domains control activity. J Biol Chem 276: 17347-17353, 2001.

15. O'Sullivan GC, Tangney M, Casey G, Ambrose M, Houston A and Barry OP: Modulation of p21-activated kinase 1 alters the behavior of renal cell carcinoma. Int J Cancer 121: 1930-1940, 2007.

16. Ching YP, Leong VY, Lee MF, Xu HT, Jin DY and Ng IO: P21-activated protein kinase is overexpressed in hepatocellular carcinoma and enhances cancer metastasis involving c-Jun NH2-terminal kinase activation and paxillin phosphorylation. Cancer Res 67: 3601-3608, 2007.

17. Li LH, Zheng MH, Luo Q, et al: P21-activated protein kinase induces colorectal cancer metastasis involving ERK activation and phosphorylation of FAK at Ser-910. Int J Oncol 37: 951-962, 2010.

18. Carter JH, Douglass LE, Deddens JA, et al: Pak-1 expression increases with progression of colorectal carcinomas to metastasis. Clin Cancer Res 10: 3448-3456, 2004.

19. Liu F, Li X, Wang C, et al: Downregulation of p21-activated kinase-1 inhibits the growth of gastric cancer cells involving cyclin B1. Int J Cancer 125: 2511-2519, 2009.

20. Sells MA, Knaus UG, Bagrodia S, Ambrose DM, Bokoch GM and Chernoff J: Human p21-activated kinase (Pak1) regulates actin organization in mammalian cells. Curr Biol 7: 202-210, 1997.

21. Ilic D, Furuta Y, Kanazawa S, et al: Reduced cell motility and enhanced focal adhesion contact formation in cells from FAK-deficient mice. Nature 377: 539-544, 1995.

22. Adam L, Vadlamudi R, Mandal M, Chernoff $\mathbf{J}$ and Kumar R: Regulation of microfilament reorganization and invasiveness of breast cancer cells by kinase dead p21-activated kinase-1. J Biol Chem 275: 12041-12050, 2000.

23. Adam L, Vadlamudi R, Kondapaka SB, Chernoff J, Mendelsohn J and Kumar R: Heregulin regulates cytoskeletal reorganization and cell migration through the p21-activated kinase-1 via phosphatidylinositol-3 kinase. J Biol Chem 273: 28238-28246, 1998.

24. Hall A: Rho GTPases and the actin cytoskeleton. Science 279: 509-514, 1998.

25. Bagrodia S and Cerione RA: Pak to the future. Trends Cell Biol 9: 350-355, 1999.

26. Vadlamudi RK, Li F, Adam L, et al: Filamin is essential in actin cytoskeletal assembly mediated by p21-activated kinase 1 . Nat Cell Biol 4: 681-690, 2002.

27. Bokoch GM, Wang Y, Bohl BP, Sells MA, Quilliam LA and Knaus UG: Interaction of the Nck adapter protein with p21-activated kinase (PAK1). J Biol Chem 271: 25746-25749, 1996. 